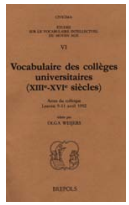




\section{THE VOCABULARY OF THE SPANISH 'COLEGIOS MAYORES' DURING THE MIDDLE AND THE MODERN AGE}

I would like to speak to you today of the vocabulary used in the Spanish colegios mayores, which can loosely be translated as greater or major colleges, from the year 1401, in which the first of them - San Bartolomé de Salamanca - was founded, until 1836, the year we can say that they disappeared. That is to say that I will allude to the basic elements of the linguistic code in force in six Spanish educational centres: The colleges of San Bartolomé (1401), Cuenca (1500), Oviedo (1517) and the El Arzobispo (1521) in Salamanca; the college of Santa Cruz (1484) in Valladolid, and the college of San Ildefonso in Alcalá de Henares (1508). The importance and influence of these institutions in the Spanish educational atmosphere of the time, I believe allows us to extend the significance of the words collected here to all the Spanish colleges of the era, except those which, as we shall see, were exclusively used and understood by the colegios mayores.

\section{The Documentary Sources}

The sources used to amass this small collegiate dictionary are of three types. Those which gave me the greatest number of facts, and the most valuable information, are of an institutional character: constitutions, statutes, ceremonies, visitation orders, etc. In this group, I mainly used the work of Luis Sala Balust titled Constituciones, Estatutos y Cerrmonias de los Colegios Seculares de la Universidad de Salamance (Salamanca, 1962-66, 4 vols.), which contains a critical edition of all these materials for the Salamancan colleges. Of a similar nature is the work of Ramón González Navarro titled Universidad Complutense. Constituciones Originales Cismerianas (Alcalá de Henares, 1984), who published the institutional documentation of the college of San Ildefonso de Alcalá de Henares. And in regard to the college of Santa Cruz de Valladolid, the study or its handbook published by M.A. Sobaler Seco, Los colegiares de Santa Cruz. Una élite de poder (Valladolid, 1987).

The second type of sources on which I based my study is in fact a manuscript: that of Francisco Pérez Bayer titled "Por la Libertad de la Literatura Española". Various copies of it survive, although in this case I used the copy in two volumes that is conserved in the Biblioteca Nacional of Madrid, manuscripts number 18.375 and 18.376. It is especially interesting in that it allows us to compare a good part of the significance of the terms that the colleges used with the different meanings used by the rest of contemporary society.

The third group is formed by various monographs. The most useful is the book of Luis Sala Balust titled Visitas y reformas de los Colegios Mayores de Salamanca en el reinado de Carlos III (Valladolid, 1958), which includes this same small collegiate dictionary (pages 427-436) compiled by F. Pérez Bayer. Sala Balust also proved useful for this study titled Reales reformas de los antiguos colegios de Salamanca anteriores a las del rinado de Carlos III (Valladolid, 1956). For Alcalá, see J. de Entrambasaguas, Grandeza y decadencia de la Universidad Complutense (Madrid, 1972). And finally, I used my own studies titled Colegios Mayores: centros de poder. Lai colegios mayores de Salamanca durante el siglo XVT (Salamanca, 1986, 3 vols.) and El Colegio Mayor de Cuenca en el siglo XVI. Estudio institucional (Salamanca, 1983).

2. Difficulties in the Determination of the Meaning of Collegiate Vocabulary.

When I began this paper my objective was to compile, in a clear and concise form, the characteristic vocabulary of the Spanish colegios mayores during the Modern Age. At that time I did not count on the enormous difficulty in translating the significance of Spanish terms that are now in disuse into another language and, especially, the translating of some words used daily in those institutions, that had between the colleges, assumed a different significance from that which they held for the rest of the contemporary Spanish society. Such are the circumstances which have conditioned the presentation of this study.

Through the work of Pérez Bayer ${ }^{1}$, we know that a specific "slang" developed within the Spanish colegios mayores, especially from the beginning of the seventeenth century onwards. Bayer states, figuratively, that the collegiate scholarships in Spain had virtually become a university faculty, and that the students who held them had to struggle to learn its specific terminology, in the same way as students of any other scientific discipline. He also warned against the custom of using many words with a different meaning than that of society at large, and to carefully

1. Especially "Por la Libertad de la Literatura Española", the manuscript of the eighteenth century already mentioned. 
refrain from using these words when people from outside the college were present. In this way, those who had not studied this dictionary were kept - and indeed are - completely confused when listening to it. And although I believe I have found the significance of some words and expressions, that both Pérez Bayer and Sala Balust confess not to understand, I am in agreement with both authors when they say not to understand the meaning of other expressions, for example: antigüedad (antiquity), moscas (flies or sparks), marqués (marquis), or when one individual yells to another: doctor, doctor, doctor, three times, to which another fellow student would respond, saying: maroma (rope). As Pérez Bayer states “... other expressions that I in truth do not understand and I believe that this happens to many who are not initiated into these mysteries, for which the author of the Genitivo ${ }^{2}$ will perhaps say that not everyone understands the language of the collegians".

This widespread use in the colleges of words or expressions with a different meaning from usual, together - as I say - with the fact that many of them belong to old Castilian, has made me give up the initial intention of translating the compiled vocabulary into another language. I consider it more illustrative to conserve it in its original form and to try to explain, as much as is possible, the meaning of the more flamboyant expressions.

\section{Characteristics of Spanish Collegiate Vocabulary}

I believe that it is possible to establish a classification of terms that collegians were using from various points of view, but I am going to refer here to only some of them. As in the other educational centres in Europe, the Spanish colleges daily used typical university terminology, which has already been compiled by other researchers. Therefore, I shall not define here words such as facultas, natio, audire, studere, lectio, gradus, ... nor many others that also belong to the collegiate linguistic code, as I consider them to be adequately explained in the works of these scholars ${ }^{3}$. 2. The so-called "author of the Genitivo" is Domingo SERRANO (the pseudonym of Manuel DOMINGUEZ SANCHEZ SALVADOR), El Genitivo de la Sierra de las Temores contra el Acusativo del Valle de Las Roncas, o un licenciado en cerro, en pelo, sin otra sociedad y por sí sólo contra un Doctor Don, Padre, Fray o Frey ..., s.l., s.i., 1760, t. II, p. 447.

3. O. WeIjERS, Vocabulaire du livre et de Pécriture au Moyen Age. (Etudes sur le vocabulaire intellectuel du Moyen Age II), Turnhout 1989. ID., Terminologie des Universités au XIIIe siècle, (Lessico Intellectuale Europeo XXXIX), Roma 1987. ID., Terminologie des Universités siecle, (Lessico Intellectuale Eure utilisé par Iinstitution nowvelle, in Missellanea Mediaevalia, 12/ naissantes. Etude sur le vocabulaire wilise par fimstion avant la lettre, in Viviarum, XXI, 1 (1983), pp. 73-82. And, with reference to Salamanca in particular, A. GarCIA Y GARCIA,
In the dictionary presented here, words are included that defined jobs, places or typically collegiate institutional ceremonies, although these at times were common to the university environment in general. In this sense words appear such as administrador, capellán, rector, capilla, estatutos or toma de puntos. From this perspective, the first thing that is noticeable is the great confusion that had to exist in the delimitation of the duties of some of the positions; there are clear overlaps between the obligations of various officials according to these same statutes.

This is especially important in the case of people who controlled economic concerns. In the college of San Bartolomé, for example, norms established the existence of such positions as sindicos, despenservos, panadero, extraordinario, receptores de la bacienda, mayordomos and contadores. Considering that the same statute limited the scholarships to a maximum of fifteen, and that economic activity was one of many that shaped the distribution of responsibilities, I very much doubt that in practice there could have existed daily such a scattered distribution of responsibilities among different people.

Another interesting group of words is that which refers to institutional ceremonies, either in the college or in the university. In alphabetical order, we find the Actos toreros, a typical Spanish term, that refers to a ceremony which is reminiscent of the old university repetitio, during which the colleges carried out all the scheduled external ceremonies, with the exception of the scientific argumentation or lecture, which at the beginning had been what was meant by this type of meeting.

The colleges failed to comply with the deeper content of some statutes, but always tried to save face by exchanging words with those which referred to them. An amusing case is the ceremony called Alooba ${ }^{4}$, which in the college of the El Arzobispo amounted to card-playing each night before a holiday, from Santa Lucia (Saint Lucy) until Semana Santa (Holy Week). It used to begin with a game called babilidades ${ }^{5}$, a term which referred to the money that the college used to give to new collegians so that, as they were not too experienced, they would lose immediately, and afterwards everyone would go to the room of the Rector to play, where people from outside the college were waiting, invited for the occasion. In the same way, in the college of Oviedo they spoke of Cabra (goat) to refer to the money with which the collegians played bolos (skittles). The most curious thing is that these games were banned in the colleges, but nobody could be accused of

Terminologia universitaria de Salamanca, in Arcbivum Latinitatis Medii Aevi (Bulletin du Cange), XLVIII-XLIX (1990), pp. 144-168.

4. Alcoba, in Spanish, usually means bedroom.

5. Habilidad, in Spanish, means capability or skill in doing anything. Notice that it has nothing to do with the meaning given to it by the colleges. 
playing them, because they were only playing Alcobas or Cabras, words that had nothing to do with either cards or skittles.

We again find this clever exchange of words when referring to women. Not only was personal contact with women banned, but the collegians could not even speak to them. In this way the members of the El Arzobispo alluded to Excusadas (excused or exempt ones) or to Trabajadoras (workers), to refer to women of disrepute in the first case, and to the artists who "worked" in theatrical plays, in the second.

In fact it was usual to break the statutes in the colleges, especially from the beginning of the seventeenth century. This led to the establishment of the expression De cajón (of the box) when speaking of the reforms that were ordered but never adhered to. They received this name because many thought that their only use was to be kept in a box.

In time, all the colleges tried to impose a system of influence in the Parliament or the Government that would permit them to assure a working future for their members. In order to do so, they began to utilize the political power of their alumni, already bureaucrats of prestige within the Spanish administration, and who therefore had a moral obligation to help them in their struggle to rise higher up in the career ladder.

To this end, the colleges developed the Bandos (factions or parties), or Tercios (thirds), different groups, who counted on a leader or Hacedor, who, from the parliament, managed the interests of a sector of collegians known as a Partido. They were assisted by the Mayor (also called cabera de partido or cabera de tercio): a collegiate guest who, from the same institution, informed the Hacedor of the wishes of the collegians. In this way we can understand the interest that the collegians had in attracting the attention of the Hacedores, to the extent that they made continual gifts to their wives (whom they called colegialas), convinced that these women could positively influence their husbands. The candidate for a collegiate scholarship who could count on the support of a Hacedor was said to have a secure sinecure (Iban a prebenda becha), because without a doubt they would soon be collegians, with the rigorous theoretical process of selection of the scholarship-holders being converted into a mere formality.

From this same perspective two other types of honorific scholarships become clear: the so-called Cartas de comensalidad and the Becas de Baño; both granted to bureaucrats or prebendaries in order to attract them to the interests or claims of the colleges. Those of de Baño were many times given to old people or even those without education, causing laughter and surprise amongst their contemporaries.

And it was the pride of feeling member of the college that obligated them to look for the best positions, up till an extreme which was known as Degradar la Beca (to degrade the scholarship) which amounted to accepting jobs of little prestige, of the type of lawyers, or beneficed priests of some small parish.

The ceremony known as Arrastre, or Arrastrar bayetas (to drag floorcloth) was very strange indeed. When the Hacedor was selecting an individual to occupy a vacant scholarship, the candidate had to enter into the college and endure all the insults and abuse that anyone wanted to throw at him. Even today this university custom survives, known by the name of novatadas (hazing).

I also collected a number of specific words in their particular "idiom", which are difficult to understand without explanation. Within this group is Cabrón, an indecent and offensive term in Spanish, which referred to the oldest freshman in the college. The Ganso (goose, in Spanish it is the same as "idiot"), in the college of Oviedo, was the sub-master of ceremonies. The comunicación de idiomas meant the lecture that the collegians did from time to time on the memorable deeds and exploits of their predecessors. Hacer la figura was the same as presenting a play. Minor colleges were known contemptuously as Picardia (which more or less corresponds to knavery). The term Sabatista meant a collegian who did not eat meat and who used the money that it would cost to buy some other type of food (or drink). The word Escalerilla (small staircase) was used with a double meaning. in Alcala it alluded to the consultation that the Royal Council used to have with the collegians of San Ildefonso in order to regulate access to the posts of the university professorship; while in Salamanca it referred to a form of punishment which applied to new collegians ${ }^{6}$. To speak of the Sigizenzas was to allude to the so-called "common areas", equivalent to our bathrooms or toilets.

Another important sector of vocabulary present is certain latin words that were used: The Cedant was a benediction that every collegian said to the memory of the founder of the college (also called $A_{m e}=$ master) when they were speaking at a university ceremony. Normally it was very long and ceremonial, in order to leave less time for the seientifie topic that was the object of their lecture. To ask for Cimilen in the College of Oviedo, meant the possibility of having dinner half an hour earlier on the nights before holidays.

But one of the more curious sayings is Ecbarse al fidelium, an allusion to the Mass of the Dead, and referring to the jobs that the least intelligent collegians aspired to: they first sat an exam to attain an

6. There also appeared other kinds of punishment, such as the so-called Pena de postes, mesa de Vaca, etc. 
ecclesiastical prebend of little importance (that they called Iglesia de primera salida - church of first exit) and, afterwards, protected by the fact that they had already been "candidates for a prebend", asked the Parliament to grant them another more important one. Along the same lines was the strange expression Praestes fides: the phrase was pronounced in a comical tone, meaning that supplementum sensuum defectui would follow. This meant that faith, an office in the Inquisition (as they interpreted the word fides), would replace the lack of wisdom and intelligence ${ }^{7}$.

I have already stated that, apart from some special words, these colleges also used the characteristic language of any other similar institution, and also, - and this is very important - they used terminology relating to purity of blood (limpieza de sangre), as we already know that these educational centres were the first to establish selective proofs for entrance into the colleges, being the staunchest defenders of the social sector of old Christians, through the expansion of the statute of purity in the Modern Age.

I now include the collegiate dictionary that I have discussed.

\section{Vocabulary of the Spanish Colegios Mayores}

\section{Actos Toreros}

Ceremonia que recordaba las antiguas repetitio universitarias salmantinas: un doctor o catedrático de propiedad subía a la cátedra en ademán de explicar una lección, quedando debajo de él un catedrático de regencia para resumir sus argumentos, pero antes de empezar la explicación, bajaba el catedrático y salían todos del aula.

\section{Administrador/Administrador Ordinario}

Colegial encargado de la administración económica de la comunidad. Es un oficio que los colegiales desempeñan de forma temporal, generalmente durante un año, $\mathrm{y}$ adquiere distintas denominaciones y cometidos dentro de cada comunidad: En el Colegio de Oviedo "es el oficio más importante, en que más cuidado debe poner el que lo tiene, por haber de pasar por sus manos toda la hacienda del colegio" (Ceremonia 23); aunque después se añade que tiene también la obligación de controlar el reparto diario de la comida (lo que equivalía al oficio del "porcionista" en el Colegio de Cuenca). Recibe el nombre de "Administrador extraordinario" el encargado de anotar el gasto diario de la casa en el Colegio de San Bartolomé; así como el que compraba

7. Interpretation of Pérez Bayer, op. cit., II, f. 501, n. 522. anualmente la tela para hacer los hábitos de los colegiales en el Colegio de Cuenca. En este mismo centro existía un "Administrador mayor" (colegial que debía llamar cada noche al administrador menor para certificar el gasto diario) y un "Administrador menor" (al cuidado de la compra del vino y del carbón).

\section{Administrador Perpetuo (vid. Patrono)}

\section{Alcobas}

Ceremonia que obligaba a jugar a los naipes a los colegiales del Colegio del Arzobispo la víspera de cada día festivo, desde Santa Lucia hasta Semana Santa. Comenzaba el acto en el comedor, donde los más nuevos debían jugar las babilidades; y perdido este dinero, se trasladaba el juego a la habitación rectoral, donde solían esperar personas de fuera especialmente invitadas para la ocasión. Una vez en la rectoria, se desarrollaba el juego al menos en dos partidas simultáneas. La que propiamente denominaban alcoba, y la llamada mesilla de vaca (el juego en una mesa adyacente en la que jugaban aquellos que no querian
arriesgar mucho dinero).

\section{Alquilones}

Universitarios que, por dinero, accedian a participar en los actos de los colegiales conclusión de Cánones y Leyes, fingiendo ser alumnos de los colegiales que presidian dichos actos.

\section{Amo}

Denominación que recibía el fundador de cada colegio cuando era nombrado por los colegiales. Se desarrolló la costumbre barroca de quitarse el bonete con gran aparatosidad, separándolo del cuerpo cuanto era posible, cada vez que se oia o pronunciaba dicho término.

\section{Arrastre/Arrastrar/Arrastrar Bayetas}

Ceremonia a la que se sometía al estudiante seleccionado para ocupar una beca vacante. El proceso comenzaba cuando el hacedor efectuaba ponían edictos como si ladiante que iba' a ocuparla. Los colegiales ponían edictos como si la plaza no estuviera otorgada de antemano, presentaba éste al Rector yido a Salamanca bajo cualquier pretexto; se desplantes que todos quisieran hacerle durates, soportando los insultos y

Bando

Documento con que se anunciaba la vacante de una beca. 
Partido, facción, nación, tercio o grupo de colegiales que defiende unos intereses particulares.

\section{Beca de Baño/Beca de Burla/Beca Formularia}

Era la que se daba en un colegio mayor a sujetos ancianos ya prebendados (canónigos, dignidades, inquisidores y hasta obispos), que no estaban en condiciones de estudiar, o que incluso jamás habían estudiado, sin que los designados se avergonzaran de las burlas a que eran sometidos al ingresar en el colegio. El objetivo de esta trasgresión constitucional consistía en atraerlos, a ellos o a sus familias, a favor de sus bandos o partidos, a fin de facilitar la consecución de los intereses colegiales.

\section{Botillero}

El colegial encargado de abastecer de agua y vino a la comunidad (Colegio de Cuenca).

\section{Cabeza de Partido/Cabeza de Tercio (vid. Mayor)}

\section{Cabras}

Medio real que se jugaba a los bolos en el Colegio de Oviedo, desde Pascua de Flores hasta el día de San Miguel.

\section{Cabrón}

El colegial más antiguo de entre los nuevos.

\section{Camarista}

En San Ildefonso de Alcalá, era un tipo de estudiante que también habitaba el colegio. Junto con los "socios" y los "estudiantes pobres de Artes", completaba el grupo de los "estudiantes pobres" que, según el capítulo XIV de las Constituciones se permitían en el centro "para mayor dilatación de la doctrina y ejercicio de los escolares". Vivían en el actual Patio de los Filósofos y, en número de trece, podían permanecer durante dos años en la institución con una sóla posibilidad de prórroga. Los camaristas recibían una limosna que equivalía a 7,4 maravedís diarios, en un momento en que se dedicaban de 30 a 34 maravedís para el mantenimiento de los colegiales. De entre ellos se elegía la figura del Vicerrector.

\section{Capellán del Colegio}

Estudiante clérigo secular elegido por los colegiales para ocupar una de las becas. Su número y consideración dentro de cada comunidad fue diferente y dependió de determinadas coyunturas, pero por lo general vivian apartados del conjunto de los colegiales, no tenian capacidad de decisión en los asuntos internos de las comunidades y eran considerados como una autoridad moral o religiosa.

Sus obligaciones pertenecían al ámbito de lo religioso y de vigilancia: la celebración de la misa diaria, los oficios divinos, la bendición de la mesa, sacar las bolas en las elecciones anuales de rector y consiliarios, el cuidado de la librería - a veces -, el rezo de las horas canónicas, ...; todo a cambio de un salario convenido. Rojas y Contreras distingue en San Bartolomé entre capellanes de manto (que viven junto a los colegiales) y capellanes de entrepuertas (cohabitando con los familiares). También entre los capellanes llamados de dentro y los de fuera, éstos últimos tenían a su cargo las misas en la capilla de la Catedral en el Colegio de San Bartolomé.

Un caso un poco especial fue el del Colegio de Oviedo, porque an constitución prescribía que todos los colegiales debian ser clérigos, al menos de primera tonsura, dos de los cuales debian ser presbiteros y desempeñar el oficio de capellanes.

\section{Capilla}

Recinto sagrado dentro de un Colegio.

Reunión o asamblea períodica de colegiales y capellanes con poder deliberativo y decisorio en todas las cuestiones relativas a la comunidad. Generalmente sólo tenían derecho a voto los colegiales y, en caso de empate, solía decidir la parte del Rector.

\section{Cartas de Comensalidad o Hermandad}

Becas honorarias que los colegiales otorgaban a los sujetos de mayor categoría del reino y especialmente a Grandes de España para atraerlos a los intereses o pretensiones colegiales. A los así designados se les enviaba una rica bandeja con la beca del colegio correspondiente.

\section{Cedant}

Alabanza oral que hacía todo colegial de la memoria de su fundador (o Amo) cada vez que un colegial se presentaba al acto de una lección de oposición, a una prebenda, a un grado o a cualquier acto universitario. Se hacía inmediatamente después de invocar la ayuda divina y solia ser muy larga con el fin de dejar menos tiempo para el tema de la propria lección u oposición.

\section{Cédula}

Papelillo o bola (de cerámica, cera o plata) que se utilizaba para emitir los votos en une elección o deliberación de Capilla. 


\section{Cédula de Vacatura (vid. Edicto de Vacatura)}

\section{Cenillas}

En el Colegio de Oviedo, día en el que no se cenaba - probablemente por prescripción religiosa de ayuno -, aunque era obligatorio asistir al comedor e incluso bendecir la mesa antes de abandonar el recinto.

\section{Ceremonias}

Conjunto normativo que regula el modo de llevarse a la práctica las actividades de la vida cotidiana, adaptándose a los sucesivos cambios de criterio de la colectividad colegial.

\section{Chofista}

Nombre que recibía también el "estudiante pobre de Artes" en Alcalá de Henares, según Entrambasaguas.

\section{Circiter}

La posibilidad de adelantar la cena media hora las vísperas de fiesta en el Colegio de Oviedo, siempre que el rector lo considerase oportuno.

\section{Colegialas}

Las esposas de los consejeros, excolegiales o hacedores, que presumían de ese título.

\section{Colegio Mayor}

Centro docente típicamente español fundado para mantener, mediante un sistema de becas, a estudiantes universitarios pobres en régimen de internado. Se caracteriza por la importancia de los privilegios de que goza, por su autonomía económica y jurisdiccional, y por requerir rigurosas condiciones físicas, intelectuales, económicas, de modo de vida y de procedencia regional a sus miembros. Inicialmente sólo hubo siete colegios mayores: El de San Clemente de Bolonia (también llamado "de los Españoles"); San Bartolomé, Cuenca, Oviedo y el Arzobispo en Salamanca; Santa Cruz en Valladolid y San Ildefonso en Alcalá de Henares. A partir del siglo XVII algunos otros centros educativos consiguen también esa denominación.

\section{Colegio Menor}

Centro docente en régimen de internado de sus becarios que se diferencia de un colegio mayor por la importancia de los privilegios y rentas de que disfrutaba, y por exigir requisitos menos rigurosos a los estudiantes aspirantes a sus becas.

\section{Coligación}

Unión avasalladora de intereses que hacían los colegios mayores en contra de las demás instituciones, especialmente contra las universidades. Esta unión se basaba en el juramento que hacían los colegiales de apoyarse sin excepción ni limitación alguna, como si de una familia se tratara, mientras estuviesen en el colegio y después de salir de él.

\section{Comunicación de Idiomas}

Lectura que solía hacerse en los colegios de las hazañas y hechos señalados llevados a cabo por sus antecesores.

\section{Comunidad}

Reunión de al menos tres colegiales (Colegio del Arzobispo).

\section{Consiliario}

Autoridad colegiada que colaboraba con el rector en la dirección y gestión de un colegio y que, como aquél, era elegida anualmente de entre todos los colegiales, de forma democrática. El procedimiento de designación era idéntico alde la rectoría y solía llevarse a cabo cines días después del nombramiento de aquél (el 16 de noviembre, dia de San Eugenio). Los consiliarios debían tener 20 años cumplidos y compartían con el rector la responsabilidad del buen funcionamiento de la casa.

\section{Constitución}

Cuerpo legislativo básico y perpétuo para la organización institucional de un colegio, redactado por el fundador o, a petición suya, por sus delegados o testamentarios.

\section{Contador}

Oficio que en el Colegio de San Bartolomé se estableció con el fin de tener cuidado de toda la hacienda del colegio.

\section{Custodio (vid. Granero)}

Dar a pistos/Dar de vez

Así se llamaba la acción de los bacedores, que otorgan las becas colegiales y, junto a ellas, la probabilidad y la aptitud para ocupar después importantes oficios eclesiásticos y civiles de la Monarquía española.

\section{De Cajón}

La repetida serie de reformas colegiales incumplidas, vino a hacerlas 
formularias, por lo que los colegiales las llamaban de cajón, no sólo por parte de los colegiales que las incumplian, sino incluso por parte de la Junta de Colegios, que las ordenaba a sabiendas de que no se iban a cumplir.

\section{Degradar la Beca}

Acabado el tiempo de la beca, aceptar un oficio que no fuera considerado como relevante o adecuado por el conjunto colegial; por ejemplo ejercer de abogado, regentar un beneficio curado, un corregimiento, etc.

\section{Despensero Menor}

Colegial encargado de llevar cuenta del gasto diario de la comunidad (Colegio de San Bartolomé). Su cometido era similar al del administrador.

\section{Diputado Anotador}

Colegial de San Ildefonso obligado semanalmente a denunciar ante el rector las faltas cometidas por los miembros de aquella universidad, así como los castigos impuestos, en lo tocante al incumplimiento de la obligación inexcusable de hablar latín dentro del recinto universitario. En compensación por su trabajo, este diputado recibía el importe de la mitad de las multas impuestas, quedando la otra mitad para los bedeles de la universidad.

\section{Diputados de Hacienda}

Dos miembros del Colegio de San Ildefonso, un colegial y el capellán mayor, que estaban obligados a exigir las cuentas económicas a todos los miembros de la comunidad, y a dar a su vez explicaciones del resultado de sus investigaciones al Claustro de la Universidad de Alcalá.

\section{Ecónomo}

Colegial encargado de recibir las rentas (Colegio de Cuenca). Cometido relacionado con el del administrador.

\section{Echarse al "Fidelium"}

Expresión tomada de la Misa de difuntos (que comenzaba con esa palabra), para referirse a la salida profesional que solían preparar los colegiales para sus compañeros menos inteligentes. Les presentaban a alguna oposición de las más sencillas, como las de las prebendas de las catedrales de Osma, Coria, Albarracín o Lugo, no con la intención de que la consiguieran, sino con el fin de que pudieran decir que habían sido opositores a una prebenda. Y con este título se presentaban después en la Corte solicitando del Real Patronato de la Camara del
Rey el nombramiento para las primeras catedrales, enfrentándose así en esta pretensión a otros opositores de gran valía.

\section{Edicto de Vacatura}

Papel que debía ponerse a la puerta del colegio, de la universidad y en otros lugares públicos, anunciando la vacante de una beca colegial. Veinte días solían tener de plazo los aspirantes para presentar su solicitud de ingreso en el colegio correspondiente y debía decidirse la elección dentro de los diez siguientes. También llamado Cédula de
vacatura.

\section{Enfermero}

Colegial a quien el rector confiaba el seguimiento de la recuperación de los posibles enfermos, obligando al familiar enfermero el cumplimiento su obligación de cuidar de ellos (Colegio de Oviedo).

\section{Escalerilla}

Consulta que hacía el Consejo Real al Colegio de San Ildefonso de Alcalá para regular los ascensos a las cátedras. Puesto que en aquella universidad no se había establecido el sistema de turno, los colegialet iban ascenciendo de las cátedras inferiores a las superiores, mediante este sistema. También se llama escalerilla a una de las mortificaciones que se aplicaba sobre los colegiales nuevos.

\section{Estatutos}

Cuerpo legislativo básico para la organización institucional de un colegio, cuyo objetivo es completar o aclarar las constituciones, adecuándolas al paso del tiempo. Frecuentemente fueron redactados por los colegiales o visitadores del colegio, aunque también existen estatutos elaborados por los propios fundadores, aclarando o matizando los artículos constitucionales.

\section{Estudiante Pobre de Artes}

Junto a los camaristas $y$ socios, estudiantes que podían alojarse en las habitaciones del Patio de Fisósofos, dentro del recinto del Colegio de San Ildefonso de Alcalá. A cambio de su trabajo en el servidio del colegio, recibían las sobras de la comida colegial más un panecillo diario. También se les llamaba chofistas.

\section{Examen de Oposición}

Ejercicio oral obligatorio para los aspirantes a una beca colegial, que se realizaba en la Capilla del Colegio en presencia de todos los colegiales, tras la Misa de Espíritu Santo. Consistía en la defensa que el opositor 
debía hacer de una cuestión elegida por él de entre tres piques practicados al azar por un familiar (vid. toma de puntos) dentro de la obra básica de la Facultad en la que desara cursar el opositor. Por eso normalmente se realizaron sobre Las Sentencias de Pedro Lombardo para los teólogos, Las Decretales para los canonistas, El Código para los juristas, y Avicena o Hipócrates para los médicos. Tras su exposición, el opositor debía responder adecuadamente a los argumentos de los colegiales, votando después éstos por cédulas secretas, bajo juramento de elegir en conciencia, al aspirante que consideraran más idóneo. En caso de empate, se seleccionaba al de mayor grado o, en último extremo, al votado por el rector (en San Bartolomé), o al que decidiera el azar.

\section{Excusadas}

Las mujeres de poca cuenta o de mala vida (Colegio Arzobispo).

\section{Familiar/Famulo}

Estudiante que disfrutaba de una beca en el colegio con la obligación de servir a la comunidad en las labores de la vida cotidiana que le fueran encomendadas, Criados del colegio, cuyo número y posibilidad de permanencia en la casa variaba de unas instituciones a otras, aunque para su ingreso tenían que someterse a similar procedimiento de selección que los colegiales, salvo que se les exigía un menor nivel de estudios realizados que a aquéllos. Debían ser de 20 años como máximo, pobres, haber estudiado tres cursos completos en cualquier facultad, ser solteros, no consanguíneos con otro miembro de la casa y que su lugar de nacimiento distara la menos cuatro leguas del de cualquier otro miembro de la comunidad. A cambio de su trabajo recibían habitación, comida - en menor cantidad que los colegiales - y, a veces, una pequeña cantidad de dinero anual.

Estaban obligados a obedecer al rector, a mantener secreto sobre los asuntos del colegio y a desempeñar el oficio que se les asignara con esmero; oficio que podía ser de despensero, capillero, refitolero, portero, enfermero, atender al rector, monaguillo, etc. Sus obligaciones se referían también a su promoción en el estudio y a la adquisición de grados universitarios. Les estaba vedada la participación en la vida colegial y generalmente eran tratados con desprecio por los colegiales.

\section{Frumentario (vid. Granero)}

\section{Ganso}

Así se llamaba en el Colegio de Oviedo al vicemaestro de ceremonias, o director de los colegiales nuevos.
Granero

El colegial encargado de cuidar del trigo en los colegios de Cuenca y San Ildefonso. En Alcalá recibía también los nombres de "custodio" o "frumentario".

\section{Habilidades}

Recibe este nombre la ceremonia que obligaba a los nuevos a jugar a los naipes dos reales que les daba el colegio. Teníalugar en el tiempo que llamaban de alcobas.

\section{Hacedor}

El que hace colegiales mayores. Excolegial - consejero o ministro - que encabeza cierto bando o partido (tercio), entre los que se repartian las vacantes de todos los colegios. Sus facultades eran en cierto modo superiores a las del Rey, puesto que se decía que daban a pistos, o daban de vez, porque a quien daban una beca, otorgaban al mismo tiempo la posibilidad de ocupar los más altos oficios de la Iglesia y el Estado. Por eso se creía que tenían más poder por ser bacedores que por ser ministros; y dado que la mayoría estaban casados, no es de extranar que sus mujeres (las colegialas) fueran muy agasajadas por los colegios, por la influencia que ejercían sobre sus maridos.

\section{Hacer la Figura}

Pequeña representación teatral que hacían los porcionistas, familiares o colegiales. La última noche de Carnavales, en el refectorio del colegio y delante de los colegiales, la realizaban los porcionistas, recibiendo en compensación una cena especial. La víspera de Pascua de Navidad bacian la figura los colegiales que aún no la habían hecho; alquilaban vestidos y todo lo necesario, y eran ayudados por familiares y porcionistas (Colegio del Arzobispo).

\section{Hortelano}

En el Colegio de Oviedo, era el colegial más antiguo que recibía el encargo del rector de controlar al empleado de la huerta. Era el único que podía entrar líbremente en ella.

\section{Hospedería}

Edificio anejo al colegio en el que podían permanecer los colegiales, tras haber concluido su tiempo de beca, a la espera del nombramiento para un empleo de su agrado.

La hospedería no se instituyó en ningún colegio mayor por constitución primitiva y fue una de las innovaciones más porfundamente contrarias al espíritu de aquéllos. Es difícil determinar el momento exacto en que 
se introdujeron, pero ya en el curso de 1548-49 (en uno de los Libros de Matrículas más antiguos de los conservados) aparece una referencia sobre la existencia de huéspedes en el Colegio de Cuenca, aunque es muy probable que la fecha de comienzo de esta costumbre sea bastante anterior. Habría que esperar hasta los estatutos de 1585 para que se legislara sobre las hospederías en el Colegio de Cuenca, y a 1591 para que se hiciera lo propio en el del Arzobispo.

\section{Iglesias de Primera Salida}

Oficios eclesiásticos a los que opositaban los colegiales por primera vez. Su presencia en una de estas oposiciones, hacía desistir de ella a los candidatos no colegiales, convencidos de la inutilidad de competir cuando probablemente el oficio estaría ya otorgado a los colegiales de antemano.

\section{Información}

Investigación que debía realizar el colegio sobre la vida, costumbres, linaje y estudios de todos los opositores a una beca colegial. Se entregaba para ello una carta de poderal colegial encargado de llevarla a cabo, que incluía siempre el interrogatorio con el que debía realizarla, el conjunto de advertencias que cada colegio tenía establecidas para ello, así como la copia de la provisión real que les autorizaba a obligar a los testigos que se negaran a declarar. Debía llevarse a cabo en el lugar o los lugares donde hubiera nacido y vivido el opositor y todos sus ascendientes. También se le llama expediente de alumno o prueba de limpieza.

\section{Interrogatorio de Abono}

Indagación mediante preguntas a testigos (testigos de abono) sobre el cumplimiento de determinadas condiciones personales - especialmente la limpieza de sangre - que se exigian a los testigos que declaraban en las informaciones de los opositores a las becas colegiales.

\section{Ir a Prebenda Hecha}

Fue muy frecuente el que los colegiales conociesen el resultado final de una oposición para ocupar un oficio antes de que se publicase la oposición al mismo; incluso antes de salir del colegio el opositor ya sabía que iba a ser elegido para desempeñarlo. En su lenguaje esto era ir a prebenda becha.

\section{Jefe}

Hacedor del colegio.

\section{Librero}

En el Colegio de Oviedo, el colegial encargado por el rector de cuidar la biblioteca. Debía evitar que se sacaran los libros de la sala, mandar a los porcionistas "regar y barrer" de cuando en cuando el suelo y limpiar el polvo a los libros; guardaba las llaves de su puerta, etc.

\section{Loables}

Refresco que solían dar el rector y los consiliarios en el momento que tomaban posesión de sus cargos.

\section{Maestro}

Título académico con que se hacían llamar todos los que ingresaban en un colegio mayor, aunque no lo hubieran adquirido en la universidad.

\section{Maestro de Ceremonias}

O preceptor de los nuevos, era el colegial más antiguo, que tenia la obligacion de enseñar las costumbres de la casa a los que iban entrande! podia también llamar la atención al rector y consiliarios si no cumplian con su cometido.

\section{Marqués}

Término usado en el argot colegial pero del que no queda constancia de su significado. Probablemente se refiera a uno de los oficiales menos cualificados de la casa. Así se hable de: "los familiares, cocinero, mozo de cocina y marqués" (Colegio del Arzobispo. Ceremonia de Alcobas, n. 15). Frecuentemente se decía la expresión "antigüedad, moscas, marqués" (Pérez Bayer).

\section{Martinetas}

Colegiales en régimen de externado existentes en los colegios de Gramática de la Universidad de Alcalá de Henares. Así llamados por la castellanización del término francés "martinets", que es como se les llamaba en los colegios de París. Estudiaban las mismas asignaturas y en las mismas horas que los colegiales internos.

\section{Mayor}

Término equivalente a cabeza de partido o cabeza de tercio. Colegial huésped más antiguo y jefe de partido, que informa regularmente de las incidencias colegiales al excolegial jefe, o hacedor, que suele vivir en la Corte. Por eso y por su enorme influencia en el bacedor, tenía asegurada la obediencia ciega de los colegiales de su partido.

\section{Mayordomo}

Colegial encargado de la hacienda, en el Colegio de San Bartolomé, 
que tenía que recibir del mayordomo de la Mancha, cada noviembre, las cuentas de las rentas que el colegio tenía en el Obispado de Cuenca.

\section{Mesa de Vaca/Mesilla de Vaca}

Mesa en la que juegan a los naipes los familiares y colegiales que no querian entrar en juegos "mayores".

\section{Mesilla/Mesilla en Seco}

Mesa del comedor reservada para quienes estuvieren cumpliendo un castigo. En el mismo sentido se habla de la mesilla en seco, en la que se sentaba el individuo castigado por el rector mientras se celebraba el acto de la comida o la cena, pero sin ingerir alimento alguno (Colegio del Arzobispo).

\section{Notario}

Todos los colegios mayores tenían que contratar un notario para que actuara en los actos o negocios que se suscitaran. En Alcalá recibía el nombre de tabelión.

\section{Ordinario}

Alimentos que constituyen la ración diaria de los miembros de la comunidad colegial.

\section{Panadero}

Colegial que debe vigilar semanalmente (Colegio de San Bartolomé) o diariamente (Colegio de San Ildefonso) la panadería del colegio. En San Ildefonso de Alcalá recibía también el nombre de pometaris.

\section{Partido mayo}

Partido, bando, tercio, o nación dominante en un colegio. En contraposición se hablaba del partido menor.

\section{Pasante}

Profesores que se dedican a ir por los colegios, posadas, y otras viviendas de estudiantes explicando aquello que no hubiera quedado claro en las clases de la universidad.

\section{Patrono}

También llamado administrador, visitador perpetuo, o protector, era una autoridad colegial cuya misión consistía en vigilar el devenir de la institución desde el exterior. Su cometido era similar al de los visitadores y se diferenciaba de ellos en que los administradores, protectores y patronos desempeñaban el oficio de por vida, en tanto que la vigilancia ejercida por los visitadores era temporal y tenía una periodicidad fija. El oficio recayó frecuentemente en un miembro de la familia del fundador y sus sucesores legales, y en contrapartida por su trabajo tenía la posibilidad de presentación de cierto número de candidatos a las becas colegiales (Colegio de Cuenca).

\section{Pena de Postes}

Una de las modalidades de castigo.

\section{Persona Soberana}

Expresion con la que se designa a los rectores de los colegios.

\section{Picardía}

Los colegios menores.

\section{Pique (vid. Toma de Puntos)}

Apertura de un libro al azar, que debía repetirse tres veces para que el opositor a una beca colegial eligiera, de entre los tres textos seleccionados, uno, para defenderlo en su examen de oposición.

\section{Pometaris (vid. Panadero)}

\section{Porcionista}

Término que se utiliza con distintas acepciones en las constituciones colegiales mayores. Generalmente se refiere al estudiante que vive en una comunidad aunque no tiene plaza de número, estando obligado a pagar una parte o porción de sus alimentos y asistencia (figura típica del Colegio de San Ildefonso de Alcalá o del Trilingüe de Salamanca). En el Colegio de San Ildefonso sus habitaciones debían estar separadas de las de los colegiales, aunque excepcionalmente alguno de los colegiales podía vivir entre ellos con objeto de ejercer funciones de vigilancia o control. También se llama porcionista al colegial nuevo que debía asistir al reparto de la comida para certificar la proporcionalidad de las raciones; o asistir los sábados por la noche al acto de pesar la carne y la fruta, certificando con su firma en el libro de despensa la fidelidad del peso. Por último (exclusivamente en el Colegio de Cuenca) hace referencia a los individuos muy pobres entre los cuales se repartirían las sobras de la comida de los colegiales; es decir, que sería el equivalente colegial a los sopistas de los conventos. 


\section{Portero}

Colegial que guarda las llaves de la puerta principal, encargándose de que se abriera y se cerrara a las horas establecidas.

\section{Praestet Fides}

Según Pérez Bayer, expresión burlesca con la que los colegiales se referían al futuro profesional de sus compañeros menos inteligentes. Se decía praestet fides entendiendo que seguía: supplementum sensuum defectui; lo que significaba que la fe, esto es, la cerrera de Inquisición (como ellos interpretaban la palabra fides) supliría la falta de sentidos (saber e inteligencia).

\section{Preceptor de los Nuevos (vid. Maestro de Ceremonias)}

\section{Prior}

En la Consticución XLVI del Colegio y Universidad de Alcalá, que trata de los cursos de licenciados en Teología, cuando se refiere a la prueba llamada "Alfonsina" que debía hacerse sin Presidente, al estilo de la Sorbona, aparece la figura de un Prior bachiller, con el encargo de presidir el ejercicio llamado del "Códice". Esta prueba consistía en recitar de memoria, tanto el Prior como el examinado, el "Códice" elegido por el alumno. Tenía gran dificultad y si no hubiera un bachiller suficientemente preparado, le podía sustituir en el cargo un licenciado o doctor en Teología. Después de esta prueba, el Prior tenía la potestad de replicar con tres nuevas conclusiones a las respuestas dadas por el examinando, medida de suma importancia si se tiene en cuenta que en los exámenes de esa universidad sólo se podía replicar con dos conclusiones. Si el Prior era bachiller, no cobraba nada por su oficio, pero si en su lugar actuaba un licenciado, éste recibía un florín de paga por su trabajo. Repitiéndose el mismo sistema de examen, el Prior también podía intervenir en la "Vespería" que era otro acto solemne.

\section{Procurador}

Colegial elegido por sus compañeros para llevar a cabo el seguimiento de los problemas judiciales del colegio (Colegio de San Bartolomé), más el cuidado de las obras de los edificios colegiales (Colegio de Oviedo). Poco gratificante debía ser este oficio, cuando la constitución obligaba a aceptarlo bajo pena de privación de la beca.

\section{Protector (vid. Patrono)}

Pruebas de Limpieza (vid. Información)

\section{Receptor}

En el Colegio de Oviedo, colegial elegido el día después de San Salvador para encargarse del gasto de la casa. Solía ser uno de los colegiales más antiguos y su cometido era similar al del administrador.

\section{Rector}

Máxima autoridad colegial, elegido anualmente de entre todos los colegiales por cédulas secretas. Las leyes imponían un acatamiento incondicional a su mandato y hasta a su propia persona. Quedaban sólo fuera de sus atribuciones tres causas criminales gravísimas: los crímenes de Lesa Majestad (atentados físicos, políticos o verbales a la figura del rey), la falsificación de moneda y los casos de herejia.

El procedimiento de la elección se llevaba a cabo generalmente el día de San Martín (11 de noviembre). Reunido el colegio en Capil. la, tras la Santa Misa y el juramento de imparcialidad, se procedía a la elección. Tenía que recaer ésta necesariamente en un miembro de la comunidad de al menos 25 años y con una antigüedad en la casa de uno a dos años, según los casos; quien se comprometia bajo jura: mento a observar y a hacer observar estrictamente las constitucioneh. Desde ese momento pasaba a ser el representante de la comunidad, conserva las llaves del arca y del edificio, cuida de que todos asistan a sus clases en la universidad, tiene que mantener la biblioteca, administrar las posesiones del colegio, tomar cuenta mensual de los gastos de la casa, dar licencia para ausentarse y castigar el incumplimiento de las leyes, en la mayoría de los casos con la ayuda y el consejo de los consiliarios.

En la legislación del Colegio de Cuenca llama la atención la extraordinaria insistencia en hacer del rectorado un cargo sobre el que recaían todos los honores, hasta el extremo de imponerse el aislamiento, de forma que el colegial que lo ocupaba quedaba totalmente sometido a su oficio. No es de extrañar que tuvieran que obligar también las leyes a aceptarlo bajo pena de privación de la beca y que los colegiales aprovecharan cualquier excusa para ausentarse en tiempos de elecciones del rector.

\section{Sabatista}

Colegial que no come carne ("grosura") y que podía cambiársele por pescado en el mismo valor económico de la carne que comían los demás compañeros (Colegio de Oviedo, Ceremonias de refectorio, $\mathrm{n}$. 61).

\section{Sacristán Mayor}

En el Colegio de San Ildefonso, el capellán con mayor autoridad. 


\section{Secretario}

Según la Ceremonia 26 de Oviedo, es elegido por cédulas secretas el día después de San Salvador y tenía que levantar acta de todas las Capillas, anotando en el margen de cada una lo que se trató en ella. Guardaba el libro en su aposento. Escribía los edictos de las prebendas, daba testimonio y contestaba al correo.

\section{Sigüenzas}

Lugares comunes (según Pérez Bayer). Actuales servicios o toilettes.

\section{Socio}

Estudiante pobre que podía residir en el Colegio de San Ildefonso, probablemente a cambio de su trabajo. Compartían habitaciones con "camaristas" y "estudiantes pobres de Artes".

\section{Receptor de la Hacienda}

Colegial o colegiales encargados de tener hecho el recuento de las operaciones financieras de la comunidad para el día de San Lucas, 18 de octubre (Colegio de San Bartolomé, Bula de Julio II - año 1507).

\section{Refectorio}

Comedor del colegio. La utilización de un término sacado de la vida monacal para referirse al ámbito secular es consecuencia de la consideración de lugares semi-monacales que los fundadores, en este caso eclesiásticos, querían dar a las fundaciones colegiales.

\section{Refitolero}

Familiar cuyo trabajo consistía en atender la mesa a la hora de las comidas de los colegiales.

\section{Renta}

Dotación económica fija que tiene una persona o una institución durante un período de tiempo determinado, normalmente un año. En los colegios representa la base de su mantenimiento económico.

\section{Síndico}

Colegial nombrado por sus compañeros para llevar las cuentas del dinero de la comunidad (Colegio de San Bartolomé).

\section{Tabelion (vid. Notario)}

\section{Tercio}

Bando, provincia, territorio, nación o partido de los varios que había en cada colegio, dirigido por un jefe (huésped) y un bacedor (excolegial que reside en la Corte) (Pérez Bayer, II, \ 27, p. 690).

\section{Toma de Puntos}

Primer acto del proceso de examen de oposición a una beca colegial. El opositor era requerido por el colegio, el cual, reunido en Capilla la víspera, con los manuales exigidos en la universidad, hacía abrir éstos por tres partes distintas - es lo que se llama dar tres piques -, dando opción de elegir uno de los tres textos al opositor para su defensa. A la mañana siguiente, tras la Misa de Espíritu Santo y el juramento de votar al candidato más idóneo, los colegiales escuchaban sus exposiciones, para posteriormente argüirle - algo similar a preguntarle - durante varias horas.

\section{Trabajador/Trabajadora}

Comediantes, artistas de teatro (Colegio del Arzobispo).

\section{Trabajos}

Comedias, obras de teatro (Colegio del Arzobispo).

\section{Trojero}

En el Colegio de Oviedo, el colegial penúltimo en antigúedad, que controlaba las entradas y salidas de pan, garbanzos y cebada; tenía que anotarlo todo en el libro correspondiente, y guardar la llave del lugar en el que se almacenaban (la "troje").

\section{Turno}

En la Universidad de Salamanca, el sistema de otorgar las cátedras por orden de antigüedad entre los becarios de los cuatro colegios mayores. De cada cinco cátedras vacantes, una era para cada colegio, y la quinta podía ser ocupada en oposición libre (Pérez Bayer, II, $\S$ 7, p. 176)

\section{Velero}

En el Colegio de Cuenca, el colegial encargado de repartir cada noche las velas.

\section{Viceconsiliario}

En el Colegio de San Ildefonso, el colegial más antiguo.

\section{Visitador}

Autoridad que ejercia como juez supremo de la disciplina en un 
colegio, cuyas decisiones adquirían categoría de ley y a cuyo mandato quedaban sometidos todos los miembros de la institución.

Hubo dos tipos de visitadores; unos eran anuales y nombrados generalmente por cada colegio para cumplir el precepto constitutional de inspección de su funcionamiento. Otros eran extraordinarios y mucho menos frecuentes, enviados por los monarcas cuando éste deseaba resolver algún problema grave.

Establecida la necesidad de su existencia por todas las constituciones fundacionales, tenían la responsabilidad de investigar todo lo relativo a la vida, costumbres, régimen de gobierno, oficios, economía y cuestiones referidas a la marcha cotidiana de un colegio; corregir los errores o resolver las dudas. Primero fueron los propios colegiales los encargados de solicitar de la institución sobre la que hubiera recaido fundacionalmente dicha responsabilidad - generalmente los cabildos catedralicios - la selección de dos beneficiados dispuestos a realizarla, pero más generalmente fueron los propios cabildos los responsables directos del nombramiento anual del visitador, que realizaba su trabajo en fechas preestablecidas.

\section{SCHOLAE, COLLEGIA ET BURSAE UNIVERSITATIS PRAGENSIS}

EIN BEITRAG ZUM WORTSCHATZ DER MITTELALTERLICHEN UNIVERSITÄTEN.

In nicht ganz drei Jahrzehnten seit seiner Gründung im Jahre 1348 entwickelte sich das Prager studium generale zu einer der meistbesuchten europäischen Universitäten ${ }^{1}$. In den achtziger Jahren des 14. Jahrhunderts bewegte sich allein an seiner selbständig gewordenen Juristenfakultit die durchschnittliche Zahl von Immatrikulationen um 140 Studenten jährlich. Einen noch höheren Durchschnitt erreichte zur gleichen Zeir die Zahl der erteilten Bakkalaureusgrade artium an der aus drei Fakultiten bestehenden Universität, wo auch Zahlen von mehr als zweihunder Promotionen jährlich keine Ausnahme bildeten. Da sich die Matrikeln dieser grösseren Universität nicht erhalten haben, kann die Gesamt frequenz des Prager studium generale nur auf Grund hypothetischer Berechnungen abgeschätzt werden. Die Zahl der Universitütsprofessoren und -studenten, die gleichzeitig in Prag weilten, wird auf 2500 bis 3000 Personen geschätzt. Noch am Beginn des 15. Jahrhunderts, da die Prager Hochschule gegen die Konkurrenz von Wien, Heidelberg, Köln, Erfurt und auch Krakau anzukämpfen hatte, betrug ihre Frequenz zumindest 1000 Personen $^{2}$. In der Agglomeration der vier Prager Städte mit einer gesamten Einwohnerzahl von vierzigtausend war eine so

1. Als knappe Einführung notiert sei hier: Peter MoRAw, Die Universitat Prag im Mittelalter. Grundyage ibrer Gescbicbte im europäischen Zusammenbang, in Die Universität zu Prag, München, 1986, pp. 9-134. Das Literaturverzeichnis bis 1975 bei Miloslava MELANOVAMichal Svatos, Bibliografie $k$ déjinám pražské univerzity do roku 1622 (=Bibliographie zur Geschichte der Prager Universität bis 1622), Praha, 1979.

2. Für eine Gesamtübersicht noch immer V.V. TOMEK, Dëje University pražské (=Geschichte der Prager Universität), Praha, 1849, pp, 311-312 und Heinz ZaTscise u. Kol., Studien zur Geschicbte der Prager Universität bis 1409, in Mitteilumgen des Verimes fur Geschicbte der Deutschen in den Sudetenländern $77(1939)$ ) 1399-1419 vgl. Tab. I-III bei František Š 77 (1939) pp. 14-28. Zuletzt für den Zeitraum obdobi 1399-1419 obdobi 1399-1419 (= Die Studentenschaft der Prager Universität vor der hussitischen Revolution), Praha, 1967 (Rozpravy ČSAV, Reihe Geisteswiss. 77-3), pp. 83-85. Hier auf pp. 16-37 eine hypothetische Durchrechnung der Gesamtfrequenz. Weiter cf. Hana VÁCLAvỨ, Poč́t graduovanjich a negraduovanjich studentri na pražské artistické fakulté y letech 1367-1398 a jejich rozdeleni podle puivodu do univerzitnich národů (=Anzahl und Herkunft der Studenten nach den Universitätsnationen an der Prager artistichen Fakultät in den Jahren 1367-1398), in Acta Universitatis Carolinae - Historia Universitatis Caroline Pragen (ferner $A U C$-HUCP) $17 / 1$ (1977) pp. 7-32 